\title{
1 Biomass and productivity of seagrasses in Africa
}

2 Michael N. Githaiga ${ }^{1,2^{*}}$, Linda Gilpin ${ }^{2}$, James. G. Kairo ${ }^{1}$, and Mark Huxham ${ }^{2}$

$3 \quad{ }^{1}$ Kenya Marine and Fisheries Research Institute, Mombasa, Kenya

$4 \quad{ }^{2}$ Edinburgh Napier University, EH11 4BN, Edinburgh, UK

5 Corresponding author: njoroge.michael04@gmail.com

6 Co-authors: huxham@napier.ac.uk, gilpin@ @apier.ac.uk, gkairo@yahoo.com

8 Abstract

9 There is growing interest in carbon stocks and flows in seagrass ecosystems, but recent

10 global reviews suggest a paucity of studies from Africa. This paper reviews work on

11 seagrass productivity, biomass and sediment carbon in Africa. Most work was

12 conducted in East Africa with a major geographical gap in West Africa. The mean

13 above-ground, below-ground and total biomasses from all studies were 174.4, 474.6 and

$14514 \mathrm{~g} \mathrm{DW} \mathrm{m}^{-2}$, respectively with a global range of 461-738 $\mathrm{g} \mathrm{DW} \mathrm{m}^{-2}$. Mean annual

15 production rate was $913 \mathrm{~g} \mathrm{DW} \mathrm{m}^{-2} \mathrm{yr}^{-1}$ (global range $816-1012 \mathrm{~g} \mathrm{DW} \mathrm{m}^{-2} \mathrm{yr}^{-1}$ ). No

16 studies were found giving sediment organic carbon, demonstrating a major gap in

17 seagrass blue carbon work. Given the small numbers of relevant papers and the large

18 geographical areas left undescribed in Africa, any conclusions remain tentative and

19 much remains to be done on seagrass studies in Africa.

20 Key words: Africa, blue carbon, productivity, seagrasses

$21 *$ *Corresponding author: njoroge.michael04@gmail.com 


\section{Introduction}

24 Understanding the role of vegetated coastal ecosystems in global carbon dynamics is a

25 field of growing interest since knowledge of natural carbon sinks and flows can

26 contribute to effective management of human impacts on the climate. Currently, our

27 understanding of the roles of different ecosystems in the global carbon budget is limited by uncertainty about, and ignorance of, both individual ecosystems and their ecological connectivity. Vegetated coastal ecosystems that, in the past, have been relatively neglected have more recently received considerable attention following the 'blue carbon' initiative, which established a clear distinction between the aquatic and terrestrial organic carbon sinks and helped to highlight the high relative efficiency of vegetated coastal sinks (Nellemann et al. 2009, http://the blue carbon initiative.org). Of the three key 'blue carbon' habitats - salt marsh, mangrove and seagrass meadows seagrasses are the most extensive but least studied. Available reviews of seagrass biomass and carbon flows globally (Duarte and Chiscano 1999, Fourqurean et al. 2012) reveal that the majority of studies have been done in Western Europe, the Mediterranean, the Caribbean, Australia and the American coasts. This is an indication of the relative paucity of information about seagrasses in African waters. Globally, seagrass ecosystems are estimated to store as much as $19.9 \mathrm{Pg}$ of organic carbon and the

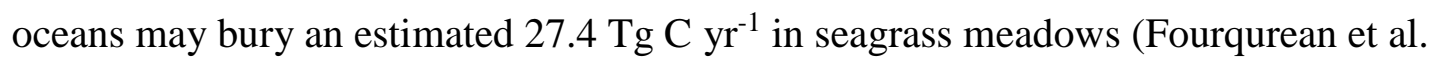

42 2012). The average standing stock of seagrass is estimated at $460 \mathrm{~g} \mathrm{DW} \mathrm{m}^{-2}$ while the average production is $5.0 \mathrm{~g} \mathrm{DW} \mathrm{m}^{-2} \mathrm{~d}^{-1}$ (Duarte and Chiscano 1999). Since these figures have been derived without much contribution from seagrass studies in Africa, estimates

45 of the global seagrass carbon budget may change substantially if sequestration and

46 storage rates in African systems are distinctive. Bearing in mind that seagrasses host a

47 high species diversity globally (Short et al. 2007) and the fact that the role of seagrasses 
48 in carbon fluxes is acknowledged (Mateo et al. 2006), there is a need to understand

49 variation in biomass and carbon storage across species and sites. The aim of the present

50 study was to carry out a comprehensive assessment of all accessible literature on

51 African seagrass species, to establish the current knowledge on biomass stocks and

52 productivity, and to identify the geographic distribution of these data around Africa.

\section{Materials and methods}

54 Both the primary and grey literature were used. Four search engines - Google Scholar,

55 Yahoo, Science Direct and ISI Web of Science - were used when looking for any

56 available information on seagrass biomass and productivity studies in Africa up to the

57 end of the year 2015. In addition, manual searches from libraries were done especially

58 for the grey literature. Several researchers thought to have been involved in seagrass

59 biomass and carbon studies in Africa were contacted to provide any available

60 information. The search terms used were 'seagrass' in combination with one of the

61 following: "above-ground biomass", "below-ground biomass", "biomass stocks",

62 "carbon burial", "productivity", "Africa", "target seagrass species" and "names of

63 countries" along the African coasts. Where data on biomass and productivity were given

64 as a range with no means reported, the mid-point was taken as an estimate of the mean

65 from that study. In some cases, relevant information was not given in the text but could

66 be reliably estimated from the figures. Data on biomass and productivity rates for

67 different species at different sites were investigated and summarized.

68 Results

69 Of the over 300 abstracts initially found, 32 papers and 8 reports or theses gave

70 information on biomass and/or productivity in Africa. Of these, 25 reported on seagrass 
71 biomass stocks alone while 15 reported entirely on productivity or a combination of

72 biomass stocks and productivity. Six reports or theses were on biomass stocks and three

73 on productivity, though one thesis reported on both biomass and productivity (Table 1).

74 Table 1: Published papers, reports/theses on seagrass biomass and productivity 75 studies around Africa

\begin{tabular}{|c|c|c|c|c|}
\hline \multirow[t]{2}{*}{ Country } & \multicolumn{2}{|l|}{ Biomass stocks } & \multicolumn{2}{|l|}{ Productivity } \\
\hline & Papers & Reports/theses & Papers & Reports/theses \\
\hline Algeria & & & Semroud 1990 & \\
\hline Egypt & $\begin{array}{l}\text { Gab-Alla } \\
2001 \\
\text { Mostafa } 1996\end{array}$ & & & \\
\hline Kenya & $\begin{array}{l}\text { Duarte et al. } \\
1998 \\
\text { Ochieng and } \\
\text { Erftemeijer } \\
1999 \\
\text { Kamermans } \\
\text { et al. } 2002 \\
\text { Ochieng and } \\
\text { Erftemeijer } \\
2003 \\
\text { Uku and } \\
\text { Björk 2005 }\end{array}$ & Gwada 2004 & $\begin{array}{l}\text { Duarte et al. } \\
1996 \\
\text { Hemminga et } \\
\text { al. } 1995 \\
\text { Ochieng and } \\
\text { Erftemeijer } \\
1999 \\
\text { Uku and Björk } \\
2005\end{array}$ & $\begin{array}{l}\text { Ochieng et } \\
\text { al.1995 }\end{array}$ \\
\hline Libya & & & $\begin{array}{l}\text { Pergent et al. } \\
2002\end{array}$ & \\
\hline Mauritania & $\begin{array}{l}\text { Laan and } \\
\text { Wolff } 2006 \\
\text { Vermaat et al. } \\
1993\end{array}$ & & $\begin{array}{l}\text { Vermaat et al. } \\
1993 \\
\text { Van Lent et al. } \\
1991\end{array}$ & \\
\hline Mauritius & Daby 2003 & & & \\
\hline
\end{tabular}




\begin{tabular}{|c|c|c|c|c|}
\hline Morocco & & $\begin{array}{l}\text { Bououraour et } \\
\text { al. } 2015 \\
\text { Boutahar et al. } \\
2015\end{array}$ & & \\
\hline Mozambique & $\begin{array}{l}\text { Bandeira } \\
1997 \\
\text { Bandeira } \\
2002 \\
\text { de Boer } 2000 \\
\text { Martins and } \\
\text { Bandeira } \\
2001 \\
\text { Paula et al. } \\
2001\end{array}$ & 更 & $\begin{array}{l}\text { Bandeira } 2002 \\
\text { de Boer } 2000\end{array}$ & $\begin{array}{l}\text { Bandeira } 2000 \\
\text { Larsson } 2009\end{array}$ \\
\hline Seychelles & Aleem 1984 & & & \\
\hline South Africa & $\begin{array}{l}\text { Adams et al. } \\
1992 \\
\text { Christie } 1981 \\
\text { Hanekom et } \\
\text { al. } 1988 \\
\text { Talbot et al. } \\
1987\end{array}$ & Grindley 1976 & & \\
\hline
\end{tabular}




\begin{tabular}{|c|c|c|c|}
\hline Tanzania & 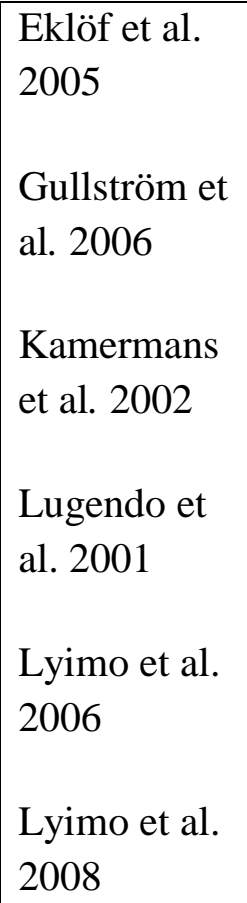 & Mvungi 2011 & $\begin{array}{l}\text { Lyimo et al. } \\
2006\end{array}$ \\
\hline Tunisia & $\begin{array}{l}\text { Sghaier et al. } \\
2011 \\
\text { Sghaier } 2012\end{array}$ & & Sghaier 2012 \\
\hline
\end{tabular}

77 These peer reviewed papers, together with the reports and theses, come from studies

78 carried out primarily on the Western Indian Ocean (WIO) coastline, especially in Kenya

79 (Gazi Bay and around Mombasa), Tanzania (sites around Zanzibar Island),

80 Mozambique (Inhaca Island), Aldabra Island in the Seychelles Republic, Mauritius and

81 along the coast of South Africa. Other studies have been conducted at Sharm El-Moyia

82 Bay along the Red Sea coastline of Egypt, Banc d' Arguin in N.W Mauritania and at

83 some bays and lagoons such as Ghar El Melh Lagoon in Northern Tunisia and at

84 Montazah Bay of Egypt on the southern Mediterranean Sea (Fig. 1). Some studies

85 (unpublished) have recently been reported from Marcha Bay, Jbel Moussa Bay and the

86 Atlantic coast of Morocco (Table 2). 


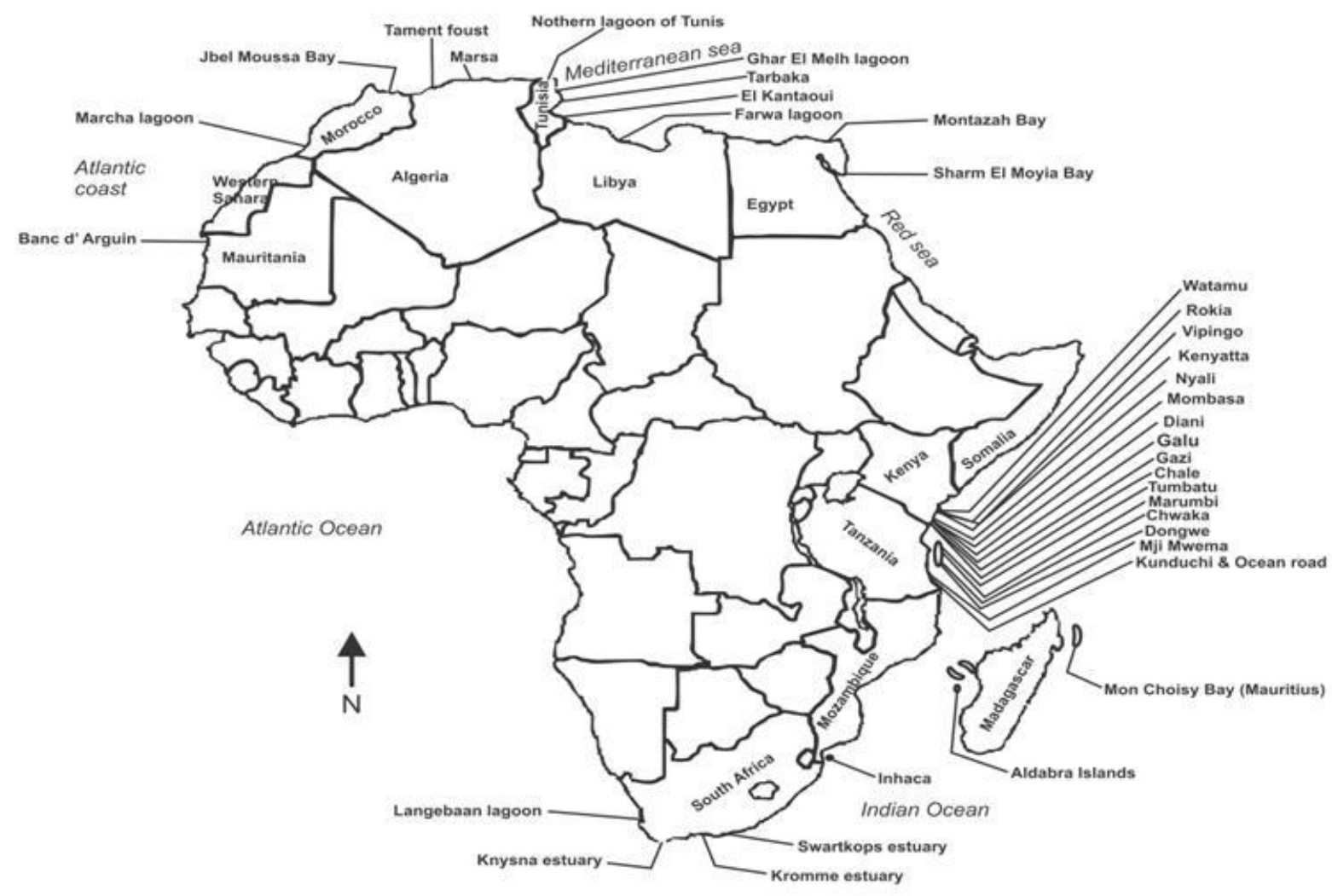

89 Fig. 1: Sites along the coastline of the African continent where seagrasses have 90 been studied 
91 Table 2: Mean ( \pm S.E) values for above-ground, below-ground and total biomass reported for different seagrass species at sites around

\section{Africa}

\begin{tabular}{|c|c|c|c|c|c|c|c|}
\hline Country & Location & $\begin{array}{l}\text { Latitude } \\
\text { \&Longitude }\end{array}$ & Species & $\begin{array}{l}\text { Above-ground } \\
\text { biomass (g DW } \\
\mathbf{m}^{-2} \text { ) }\end{array}$ & $\begin{array}{l}\text { Below -ground } \\
\text { biomass (g DW m²) }\end{array}$ & $\begin{array}{l}\text { Total biomass (g } \\
\text { DW } \mathrm{m}^{-2} \text { ) }\end{array}$ & Reference \\
\hline \multirow[t]{2}{*}{ Egypt } & Montazah Bay & $\begin{array}{l}31^{\circ} 12^{\prime} \mathrm{N}, \\
29^{\circ} 55^{\prime} \mathrm{E}\end{array}$ & Cymodocea nodosa & 287 & & & Mostafa 1996 \\
\hline & $\begin{array}{l}\text { Sharm El Moyia } \\
\text { Bay }\end{array}$ & $\begin{array}{l}27^{\circ} 9^{\prime} \mathrm{N} \\
34^{\circ} 3^{\prime} \mathrm{E}\end{array}$ & Halophila stipulaceae & & & 270 & Gab-Alla 2001 \\
\hline \multirow[t]{5}{*}{ Kenya } & Galu & $4^{\circ} 18^{\prime} \mathrm{S}, 39^{\circ} 32^{\prime} \mathrm{E}$ & $\begin{array}{l}\text { Thalassodendron } \\
\text { ciliatum }\end{array}$ & & & $40.6 \pm 40.6$ & Uku et al. 1996 \\
\hline & Diani & $4^{\circ} 18^{\prime} \mathrm{S}, 10^{\circ} 32^{\prime} \mathrm{E}$ & $\begin{array}{l}\text { Thalassodendron } \\
\text { ciliatum }\end{array}$ & & & $279.3 \pm 97.6$ & Uku et al. 1996 \\
\hline & Diani & $4^{\circ} 18^{\prime} \mathrm{S}, 10^{\circ} 32^{\prime} \mathrm{E}$ & Mixed & & & $430(33)$ & $\begin{array}{l}\text { Kamermans et } \\
\text { al. } 2002\end{array}$ \\
\hline & Gazi & $4^{\circ} 25^{\prime} \mathrm{S}, 39^{\circ} 30^{\prime} \mathrm{E}$ & $\begin{array}{l}\text { Thalassodendron } \\
\text { ciliatum }\end{array}$ & $316.1 \pm 41$ & $368.1 \pm 22$ & $725.5 \pm 252.5$ & $\begin{array}{l}\text { Ochieng and } \\
\text { Erftemeijer } 2003\end{array}$ \\
\hline & Chale lagoon & $4^{\circ} 25^{\prime} \mathrm{S}, 39^{\circ} 30^{\prime} \mathrm{E}$ & $\begin{array}{l}\text { Thalassodendron } \\
\text { ciliatum }\end{array}$ & & 243.4 & & $\begin{array}{l}\text { Duarte et al. } \\
1998\end{array}$ \\
\hline
\end{tabular}




\begin{tabular}{|c|c|c|c|c|c|c|c|}
\hline & Mombasa & $4^{\circ} 2^{\prime} \mathrm{S}, 39^{\circ} 41^{\prime} \mathrm{E}$ & Mixed & & & $471.6 \pm 66.7$ & $\begin{array}{l}\text { Kamermans et } \\
\text { al. } 2002\end{array}$ \\
\hline & Roka & $1^{\circ} 36^{\prime} \mathrm{S}, 39^{\circ} 12^{\prime} \mathrm{E}$ & Mixed & & & $644(7)$ & $\begin{array}{l}\text { Kamermans et } \\
\text { al. } 2002\end{array}$ \\
\hline & Mombasa & $4^{\circ} 2^{\prime} \mathrm{S}, 39^{\circ} 41^{\prime} \mathrm{E}$ & Mixed & & & $760 \pm 96$ & Ochieng and \\
\hline & Marine Park & & & & & & Erftemeijer 1999 \\
\hline & Nyali & $4^{\circ} 03^{\prime} \mathrm{S}, 39^{\circ} 43^{\prime} \mathrm{E}$ & $\begin{array}{l}\text { Thalassodendron } \\
\text { ciliatum-North East } \\
\text { monsoon }\end{array}$ & $277.4 \pm 36.3$ & $364.9 \pm 83.5$ & & Gwada 2004 \\
\hline & & & $\begin{array}{l}\text { Thalassodendron } \\
\text { ciliatum-South East } \\
\text { monsoon }\end{array}$ & $269.5 \pm 65$ & $312.0 \pm 123$ & & \\
\hline & Nyali & $4^{\circ} 03^{\prime} \mathrm{S}, 39^{\circ} 43^{\prime} \mathrm{E}$ & Mixed & & & $604(33)$ & $\begin{array}{l}\text { Kamermans et } \\
\text { al. } 2002\end{array}$ \\
\hline & Kenyatta & $4^{\circ} 00^{\prime} \mathrm{S}, 39^{\circ} 44^{\prime} \mathrm{E}$ & Mixed & & & $233(33)$ & \\
\hline & Watamu & $3^{\circ} 23^{\prime} \mathrm{S}, 39^{\circ} 59^{\prime} \mathrm{E}$ & Mixed & & & $457(33)$ & \\
\hline Mauritania & Banc d' Arguin & $\begin{array}{l}20^{\circ} 35^{\prime} \mathrm{N} \\
16^{\circ} 15^{\prime} \mathrm{W}\end{array}$ & Mixed & & & 335 & $\begin{array}{l}\text { Vermaat et al. } \\
1993\end{array}$ \\
\hline & & & Mixed & & 255.0 & & $\begin{array}{l}\text { Laan and Wolff } \\
2006\end{array}$ \\
\hline Mauritius & $\begin{array}{l}\text { Mon Choisy } \\
\text { Bay }\end{array}$ & $20^{\circ} 17^{\prime} \mathrm{S}, 5733^{\prime} \mathrm{W}$ & $\begin{array}{l}\text { Syringondium } \\
\text { isoetifolium }\end{array}$ & & & 129.3 & Daby 2003 \\
\hline
\end{tabular}




\begin{tabular}{|c|c|c|c|c|c|c|c|}
\hline & & & Halophila ovalis & & & 102.5 & \\
\hline \multirow[t]{6}{*}{ Morocco } & Marcha lagoon & $40^{\circ} 39^{\prime} \mathrm{N}, 8^{\circ} 48^{\prime} \mathrm{W}$ & Cymodocea nodosa & $8.02-61.2$ & $10.8-235$ & & Boutahar et al. \\
\hline & & & & & & & 2015 \\
\hline & Atlantic coast & $23^{\circ} 30^{\prime} \mathrm{N}$ & Zostera noltii & 32- 259 & $21-314$ & & Bououarour et \\
\hline & & $15^{\circ} 56^{\prime} \mathrm{W}$ & & & & & al. 2015 \\
\hline & Jbel Moussa & $30^{\circ} 8^{\prime} \mathrm{N}, 5^{\circ} 21^{\prime} \mathrm{W}$ & Zostera noltii & $3.08 \pm 1.12$ & & $7.72 \pm 1.38$ & \\
\hline & Bay & & & & & & \\
\hline \multirow[t]{13}{*}{ Mozambique } & Inhaca & $25^{\circ} 58^{\prime} \mathrm{S}, 32^{\circ} 55^{\prime} \mathrm{E}$ & Thalassodendron & $355.2 \pm 111.1$ & $792.4 \pm 342.9$ & $1148(30)$ & Bandeira 1997 \\
\hline & & & Zostera capensis & $15.7 \pm 4.5$ & $173.4 \pm 47.5$ & $190 \pm 51.2(10)$ & de Boer 2000 \\
\hline & & & (Summer) & & & & \\
\hline & & & Cymodocea serrulata & $34.1 \pm 18.6$ & $38.6 \pm 14.0$ & $82.0 \pm 30.8(10)$ & \\
\hline & & & (Summer) & & & & \\
\hline & & & Halodule wrightii & $16.0 \pm 22.2$ & $17.1 \pm 14.5$ & $22.2 \pm 21.7(10)$ & \\
\hline & & & (Summer) & & & & \\
\hline & & & Zostera capensis & $25.7 \pm 8.0$ & $198.9 \pm 75$ & $219.5 \pm 78.1(10)$ & \\
\hline & & & (Winter) & & & & \\
\hline & & & Cymodocea serrulata & $17.6 \pm 15.2$ & $27.0 \pm 14.4$ & $43.1 \pm 21.8(10)$ & \\
\hline & & & (Winter) & & & & \\
\hline & & & Halodule wrightii & $6.9 \pm 5.5$ & $18.1 \pm 6.5$ & $22.9 \pm 8.2(10)$ & \\
\hline & & & (Winter) & & & & \\
\hline
\end{tabular}




\begin{tabular}{|c|c|c|c|c|c|c|c|}
\hline & $\begin{array}{l}\text { Inhaca } \\
\text { (Northern Bay) }\end{array}$ & $25^{\circ} 58^{\prime} \mathrm{S}, 32^{\circ} 55^{\prime} \mathrm{E}$ & Thalassia hemprichii & $154.4 \pm 22.7$ & $633.0 \pm 163.5$ & $787.4 \pm 233.8$ & $\begin{array}{l}\text { Martins and } \\
\text { Bandeira } 2001\end{array}$ \\
\hline & & & $\begin{array}{l}\text { Halodule wrightii } \\
\text { Halophila ovalis } \\
\text { Zostera capensis } \\
\text { Cymodocea rotundata }\end{array}$ & & & $\begin{array}{l}30.7 \pm 11.9 \\
0.6 \pm 0.4 \\
4.8 \pm 2 \\
39.9 \pm 18.7\end{array}$ & \\
\hline & $\begin{array}{l}\text { Inhaca } \\
\text { (Southern Bay) }\end{array}$ & $25^{\circ} 58^{\prime} \mathrm{S}, 32^{\circ} 55^{\prime} \mathrm{E}$ & Thalassia hemprichii & $147.1 \pm 68.65$ & $1729.7 \pm 495.25$ & $1876 \pm 389.4$ & \\
\hline & & & $\begin{array}{l}\text { Halodule wrightii } \\
\text { Halophila ovalis } \\
\text { Zostera capensis } \\
\text { Cymodocea rotundata }\end{array}$ & & & $\begin{array}{l}0.9 \pm 0.7 \\
0 \pm 0 \\
0 \pm 0 \\
4.5 \pm 43\end{array}$ & \\
\hline & & $25^{\circ} 58^{\prime} \mathrm{S}, 32^{\circ} 55^{\prime} \mathrm{E}$ & $\begin{array}{l}\text { Thalassodendron } \\
\text { ciliatum } \\
\text { Thalassia hemprichii } \\
\text { Zostera capensis }\end{array}$ & $\begin{array}{l}50.1-170.7 \\
14.2-291.1 \\
7.9-51.3\end{array}$ & $\begin{array}{l}0.04-1471.1 \\
9.21-1307.6 \\
66.0-195.5\end{array}$ & & Paula et al. 2001 \\
\hline Seychelles & $\begin{array}{l}\text { Inhaca } \\
\text { Aldabra Island }\end{array}$ & $\begin{array}{l}25^{\circ} 58^{\prime} \mathrm{S}, 32^{\circ} 55^{\prime} \mathrm{E} \\
9^{\circ} 41^{\prime} \mathrm{S}, 46^{\circ} 42^{\prime} \mathrm{E}\end{array}$ & $\begin{array}{l}\text { Thalassia hemprichii } \\
\text { Halodule uninervis } \\
\text { Halophila ovalis }\end{array}$ & $49.8 \pm 3.1$ & & $\begin{array}{l}243 \\
46.5\end{array}$ & $\begin{array}{l}\text { Larsson } 2009 \\
\text { Aleem } 1984\end{array}$ \\
\hline
\end{tabular}




\begin{tabular}{|c|c|c|c|c|c|c|c|}
\hline & & & Mixed species & & & 425 & \\
\hline & & & Thalassia hemprichii & & & 412.5 & \\
\hline & & & Thalassodendron & & & 468 & \\
\hline & & & ciliatum & & & & \\
\hline & & & Syringondium & & & 435 & \\
\hline & & & isoetifolium & & & & \\
\hline \multirow[t]{11}{*}{ South Africa } & Knysna estuary & $34^{\circ} 05^{\prime} \mathrm{S}, 23^{\circ} 21^{\prime} \mathrm{E}$ & Zostera capensis & 206 & & & Grindley 1976 \\
\hline & Langebaan & $33^{\circ} 01^{\prime} \mathrm{S}, 18^{\circ} 01^{\prime} \mathrm{E}$ & Zostera capensis & 217 & & & Christie 1981 \\
\hline & lagoon & & & & & & \\
\hline & Swartkops & $33^{\circ} 52^{\prime} \mathrm{S}, 25^{\circ} 38^{\prime} \mathrm{E}$ & Zostera capensis & & & $75.8-124.7$ & Talbot et al 1987 \\
\hline & estuary & & & & & & \\
\hline & Kromme & $34^{\circ} 09^{\prime} \mathrm{S}, 24^{\circ} 51^{\prime} \mathrm{E}$ & Zostera capensis & $105 \pm 44$ & & & Hanekom et al. \\
\hline & Estuary & & (Winter 1979) & & & & 1988 \\
\hline & & & Zostera capensis & $55 \pm 21$ & & & \\
\hline & & & (Summer 1980) & & & & \\
\hline & Kromme & $34^{\circ} 09^{\prime} \mathrm{S}, 24^{\circ} 51^{\prime} \mathrm{E}$ & & & & 244 & Adams et \\
\hline & Estuary & & & & & & al.1992 \\
\hline \multirow[t]{6}{*}{ Tanzania } & Chwaka & $6^{\circ} 10^{\prime} \mathrm{S}, 39^{\circ} 26^{\prime} \mathrm{E}$ & Thalassia hemprichii & $897.2 \pm 754.8$ & - & - & Kamermans et \\
\hline & & & & & & & al. 2002 \\
\hline & Chwaka & $6^{\circ} 10^{\prime} \mathrm{S}, 39^{\circ} 26^{\prime} \mathrm{E}$ & Thalassia hemprichii & & & 85 & Eklöf et al. 2005 \\
\hline & & & Enhalus acoroides & & & 100 & \\
\hline & & & Thalassodendron & & & 90 & \\
\hline & & & ciliatum & & & & \\
\hline
\end{tabular}




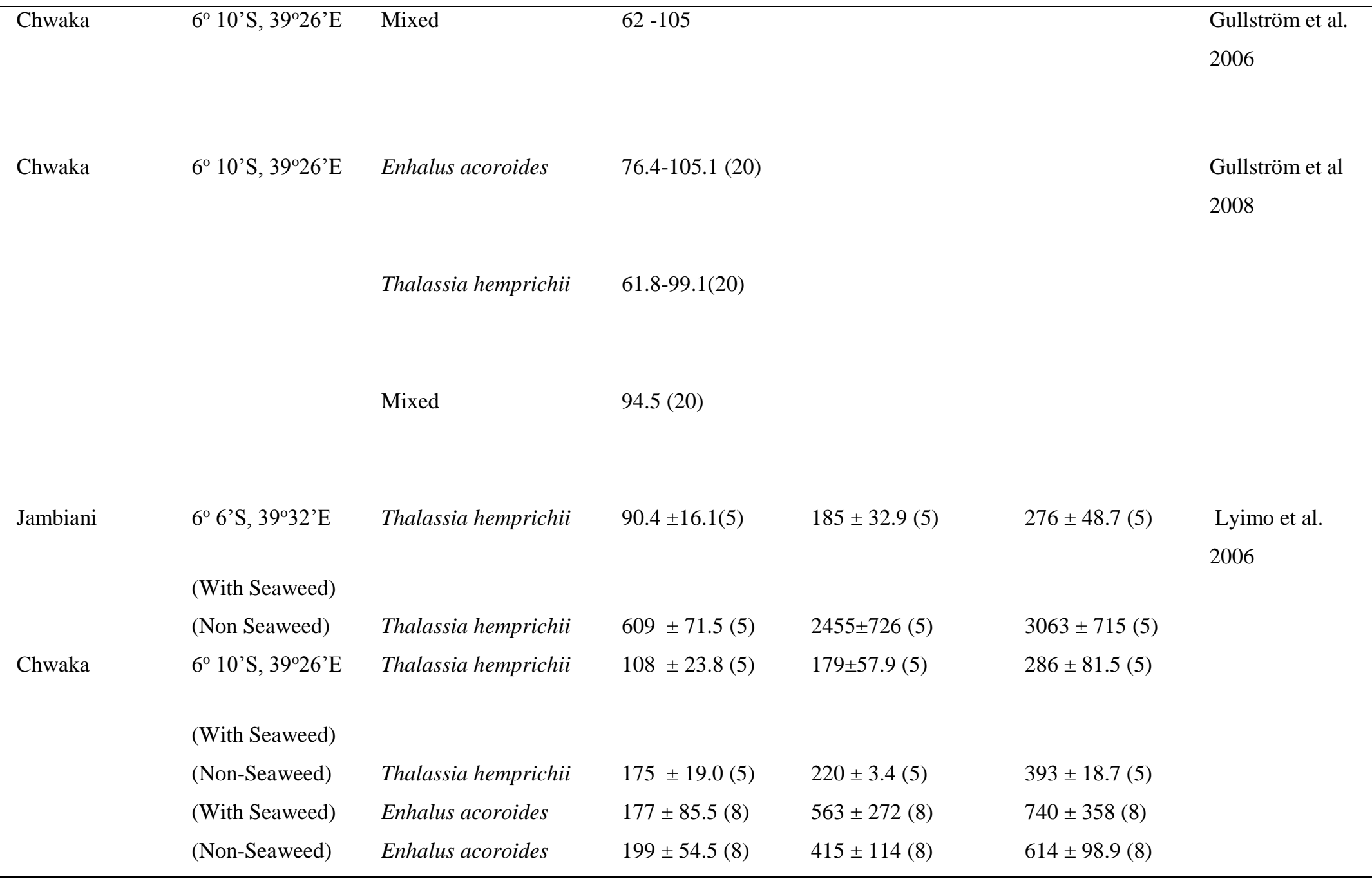




\begin{tabular}{|c|c|c|c|c|c|c|}
\hline \multirow[t]{5}{*}{ Marumbi } & $6^{\circ} 13^{\prime} \mathrm{S}, 39^{\circ} 28^{\prime} \mathrm{E}$ & Thalassia hemprichii & $465 \pm 183(5)$ & $904 \pm 129(5)$ & $1369 \pm 266(5)$ & \\
\hline & (With Seaweed) & & & & & \\
\hline & (Non-Seaweed) & Thalassia hemprichii & $301 \pm 42.1(5)$ & $442 \pm 66.9(5)$ & $742 \pm 81(5)$ & \\
\hline & (With Seaweed & Enhalus acoroides & $144 \pm 63.0(8)$ & $810 \pm 356(8)$ & $953 \pm 418(8)$ & \\
\hline & (Non-Seaweed) & Enhalus acoroides & $143 \pm 57.5(8)$ & $512 \pm 207(8)$ & $655 \pm 264(8)$ & \\
\hline \multirow[t]{4}{*}{ Chwaka } & $6^{\circ} 10^{\prime} \mathrm{S}, 39^{\circ} 26^{\prime} \mathrm{E}$ & Mixed & & & $142.4-1652$ & Lyimo et al. \\
\hline & & & & & & 2008 \\
\hline & (With Seaweed) & & & & & \\
\hline & (Non-Seaweed) & & & & 212.9-1829 & \\
\hline \multirow[t]{3}{*}{ Jambiani } & $6^{\circ} 6^{\prime} \mathrm{S}, 39^{\circ} 32^{\prime} \mathrm{E}$ & mixed & & & $880.4-3467$ & \\
\hline & (With Seaweed) & & & & & \\
\hline & (Non-Seaweed) & & & & 203.4-3810 & \\
\hline Kunduchi\& & $6^{\circ} 40^{\prime} \mathrm{S}, 39^{\circ} 13^{\prime} \mathrm{E}$ & Mixed & & & $0.25-135.29$ & Lugendo et al. \\
\hline Ocean road & & & & & & 2001 \\
\hline \multirow[t]{2}{*}{ Ocean road } & $6^{\circ} 45^{\prime} \mathrm{S}, 39^{\circ} 20^{\prime} \mathrm{E}$ & Thalassia hemprichii & $307.0 \pm 74.9$ & $412.1 \pm 93.3$ & & Mvungi 2011 \\
\hline & & Cymodocea serrulata & $202.7 \pm 69.6$ & $267.7 \pm 147.9$ & & \\
\hline \multirow[t]{2}{*}{ Mji-mwema } & $6^{\circ} 38^{\prime} \mathrm{S}, 39^{\circ} 40^{\prime} \mathrm{E}$ & Thalassia hemprichii & $267.0 \pm 43.8$ & $1177.4 \pm 265.2$ & & \\
\hline & & Cymodocea serrulata & $352.2 \pm 141.7$ & $737.2 \pm 260.8$ & & \\
\hline
\end{tabular}




\begin{tabular}{|c|c|c|c|c|c|c|c|}
\hline & Kiwengwa & $5^{\circ} 60^{\prime} \mathrm{S}, 39^{\circ} 23^{\prime} \mathrm{E}$ & Mixed & & & $115(30)$ & Kamermans et \\
\hline & Dongwe & $6^{\circ} 11^{\prime} \mathrm{S}, 39^{\circ} 32^{\prime} \mathrm{E}$ & Mixed & & & $224(21)$ & al. 2002 \\
\hline \multirow[t]{4}{*}{ Tunisia } & Ghar El Melh & $37^{\circ} 09^{\prime} \mathrm{N}$ & Cymodocea nodosa & $97.3 \pm 51.4$ & $264.7 \pm 69.2$ & $327.7 \pm 86.1$ & Sghaier 2012 \\
\hline & Lagoon & $10^{\circ} 13^{\prime} \mathrm{E}$ & Cymodocea nodosa & $82.5 \pm 15.38$ & $333.9 \pm 49.4$ & $413.8 \pm 46$ & $\begin{array}{l}\text { Sghaier et al. } \\
2011\end{array}$ \\
\hline & Northern lagoon & $37^{\circ} 14^{\prime} \mathrm{N}, 09^{\circ}$ & Zostera noltii & & & 79.75 & Imen et al. 2014 \\
\hline & of Tunis & $56^{\prime} \mathrm{E}$ & & & & & \\
\hline
\end{tabular}

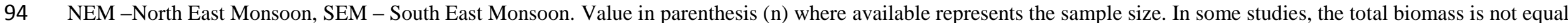

95 to the sum of the above-ground and the below-ground due to differences in the samples sizes but are captured as reported in the studies.

96 The four families of seagrass and species studied on biomass and productivity in Africa; Hydrocharitaceae (Enhalus acoroides (L.F) Royle, Halophila minor (Zoll.) den

97 Hartog, Halophila ovalis (R.Br.) Hook f., Halophila stipulaceae (Forsk.) Aschers and Thalassia hemprichii (Enhrenberg) Ascherson) Cymodoceae (Cymodocea rotundata

98 Ehrenb. Et Hempx.et Aschers.Cymodocea serrulata (R.Br.) Aschers. et Magnus, Cymodocea nodosa (Ucria) Aschers., Halodule uninervis (Forsk.) Aschers. in Bossier,

99 Halodule wrightii Aschers., Syringondium isoetifolium (Aschers.) Dandy and Thalassondendron ciliatium (Forsk.) den Hartog); Zosteraceae (Zostera capensis Setchell,

100 Zostera noltii, ); Posidonaceae (Posidonia oceanica (L.) Delile). 
In press with Botanica Marina, 2016

102 Data were available for 14 species, with biomass data available for 13 species (Table 2), 103 while data on seagrass productivity were available for 10 species (Table 3). Most of the 104 seagrass biomass studies considered mixed stands, but Thalassodendron ciliatum and 105 Thalassia hemprichii were the most widely studied individual species, each having been 106 a subject of research in 9 out of the 35 locations where biomass studies were reported 107 and in 5 and 6 locations, respectively, out of the 18 locations for productivity studies. 108 Halodule wrightii, Cymodocea rotundata, Halophila stipulaceae and Halodule 109 uninervis have been studied for biomass stocks in only one location each. Similarly, 110 with the exception of Thalassia hemprichii and Thalassodendron ciliatum, a majority of 111 the other species reported in productivity research were studied in only one location 112 (Table 3). Thalassodendron ciliatum was the only species reported to have been studied 113 for all the productivity indices (Table 3). 
Table 3: Productivity values expressed as rates of leaf growth, leaf dry weight production, rhizome growth and total dry weight production for different seagrass species at sites around Africa

116

\begin{tabular}{|c|c|c|c|c|c|c|c|c|c|}
\hline Country & location & $\begin{array}{l}\text { Latitude \& } \\
\text { Longitude }\end{array}$ & Species & Season & $\begin{array}{l}\begin{array}{l}\text { Leaf } \\
\text { growth } \\
(\mathrm{mm}\end{array} \\
\text { shoot }^{-1} \\
\left.\text { day }^{-1}\right)\end{array}$ & $\begin{array}{l}\text { Leaf } \\
\text { production } \\
(\mathrm{g} \text { DW } \\
\left.\text { shoot }^{-1} \mathbf{d}^{-1}\right)\end{array}$ & $\begin{array}{l}\text { Rhizome } \\
\text { growth }(\mathbf{m m} \\
\left.\mathbf{d}^{-1}\right)\end{array}$ & $\begin{array}{l}\text { Total } \\
\text { Production } \\
(\mathrm{g} \text { DW m } \\
\left.\mathbf{d}^{-1}\right)\end{array}$ & Reference \\
\hline \multirow[t]{2}{*}{ Algeria } & Marsa & $35^{\circ} 51^{\prime} \mathrm{N}, 10^{\circ} 35^{\prime} \mathrm{E}$ & Posidonia oceanica & & & & 0.02 & & Semroud 1990 \\
\hline & Tament foust & & & & & & 0.35 & & \\
\hline \multirow[t]{9}{*}{ Kenya } & Gazi Bay & $4^{\circ} 25^{\prime} \mathrm{S}, 39^{\circ} 30^{\prime} \mathrm{E}$ & $\begin{array}{l}\text { Thalassodendron } \\
\text { ciliatum }\end{array}$ & & & & & 7.5 & $\begin{array}{l}\text { Hemminga et al. } \\
1995\end{array}$ \\
\hline & & $4^{\circ} 25^{\prime} \mathrm{S}, 39^{\circ} 30^{\prime} \mathrm{E}$ & $\begin{array}{l}\text { Thalassodendron } \\
\text { ciliatum }\end{array}$ & & $20.7 \pm 0.8$ & & & $4.43 \pm 2.7$ & Ochieng 1995 \\
\hline & & $4^{\circ} 25^{\prime} \mathrm{S}, 39^{\circ} 30^{\prime} \mathrm{E}$ & Mixed species & & & & & $2.4 \pm 0.6$ & Ochieng 1995 \\
\hline & Chale lagoon & $4^{\circ} 25^{\prime} \mathrm{S}, 39^{\circ} 30^{\prime} \mathrm{E}$ & $\begin{array}{l}\text { Thalassodendron } \\
\text { ciliatum }\end{array}$ & & & & 0.4 & & Duarte et al. 1996 \\
\hline & $\begin{array}{l}\text { Mombasa } \\
\text { Marine park }\end{array}$ & $4^{\circ} 2^{\prime} \mathrm{S}, 39^{\circ} 41^{\prime} \mathrm{E}$ & Mixed species & & & & & $8.2 \pm 2.8$ & $\begin{array}{l}\text { Ochieng and } \\
\text { Erftemeijer } 1999\end{array}$ \\
\hline & Nyali & $4^{\circ} 03^{\prime} \mathrm{S}, 39^{\circ} 43^{\prime} \mathrm{E}$ & Thalassia hemprichii & S.E & $17.2 \pm 9.5$ & $0.008 \pm$ & & $5.5 \pm 4.9$ & Uku and Björk \\
\hline & & & & & & 0.002 & & (30) & 2005 \\
\hline & & & & N.E & $28.5 \pm 4.1$ & $0.008 \pm$ & & $5.3 \pm 0.5$ & \\
\hline & & & & & & 0.006 & & (30) & \\
\hline
\end{tabular}




\begin{tabular}{|c|c|c|c|c|c|c|c|c|c|}
\hline & Vipingo & $3^{\circ} 45^{\prime} \mathrm{S}, 39^{\circ} 50^{\prime} \mathrm{E}$ & & S.E & $17.1 \pm 2.6$ & $0.004 \pm$ & & $2.4 \pm 1.04$ & \\
\hline & & & & & & 0.001 & & (30) & \\
\hline & & & & N.E & $17.1 \pm 2.8$ & $0.004 \pm$ & & $3.3 \pm 1.1(30)$ & \\
\hline & & & & & & 0.002 & & & \\
\hline & Nyali & $4^{\circ} 03^{\prime} \mathrm{S}, 39^{\circ} 43^{\prime} \mathrm{E}$ & Thalassodendron & S.E & $17.3 \pm 1.6$ & $0.005 \pm$ & & $3.7 \pm 2.4$ & \\
\hline & & & ciliatum & & & 0.005 & & (30) & \\
\hline & & & & N.E & $18.8 \pm 5.9$ & $0.006 \pm$ & & $3.1 \pm 1.8$ & \\
\hline & & & & & & 0.003 & & (30) & \\
\hline & Vipingo & $3^{\circ} 45^{\prime} \mathrm{S}, 39^{\circ} 50^{\prime} \mathrm{E}$ & & S.E & $12.4 \pm 5.7$ & $0.005 \pm$ & & $2.9 \pm 2.4$ & \\
\hline & & & & & & 0.002 & & (30) & \\
\hline & & & & N.E & $12.4 \pm 5.3$ & $0.004 \pm$ & & $1.8 \pm 1.6$ & \\
\hline & & & & & & 0.001 & & (30) & \\
\hline & Nyali & $4^{\circ} 03^{\prime} \mathrm{S}, 39^{\circ} 43^{\prime} \mathrm{E}$ & Cymodocea & S.E & $12.8 \pm 1.6$ & $0.002 \pm$ & & $2.1 \pm 0.5$ & \\
\hline & & & rotundata & & & 0.0005 & & (30) & \\
\hline & & & & N.E & $14.9 \pm 1.8$ & $0.002 \pm$ & & $2.3 \pm 0.5$ & \\
\hline & & & & & & 0.0002 & & (30) & \\
\hline & Vipingo & $3^{\circ} 45^{\prime} \mathrm{S}, 39^{\circ} 50^{\prime} \mathrm{E}$ & & S.E & $10.0 \pm 9.1$ & $0.001 \pm$ & & $2.0 \pm 1.1$ & \\
\hline & & & & & & 0.0006 & & (30) & \\
\hline & & & & N.E & $11.7 \pm 2.0$ & $0.001 \pm$ & & $1.9 \pm 1.0$ & \\
\hline & & & & & & 0.0005 & & (30) & \\
\hline Libya & Farwa lagoon & $33^{\circ} 05^{\prime} \mathrm{N}, 11^{\circ} 44^{\prime} \mathrm{E}$ & & & & & $0.02-0.1$ & & Pergent et al. 2002 \\
\hline Mauritania & Banc d Arguin & $20^{\circ} 35^{\prime} \mathrm{N}, 16^{\circ} 15^{\prime} \mathrm{W}$ & Cymodocea nodosa & & & & & 0.003 & Van Lent et al. \\
\hline & & & & & & & & & 1991 \\
\hline
\end{tabular}




\section{Zostera noltii}

$25^{\circ} 58^{\prime} \mathrm{S}, 32^{\circ} 55^{\circ} \mathrm{E}$

Mozambiq

ue

Inhaca Island

(1)

Thalassodendron

ciliatum

Thalassodendron

ciliatum

Zostera capensis

Zostera capensis

Cymodocea

serrulata

Cymodocea

serrulata

Halodule wrightii

Thalassia hemprichii

34 09'S, 2451'E Zostera. capensis

South

Kromme

estuary

Tanzania

Thalassia hemprichii

\section{Chwaka}

$6^{\circ} 10^{\prime} \mathrm{S}, 39^{\circ} 26^{\prime} \mathrm{E}$

Jambiani

$\begin{array}{lll}\text { Summer } & 1.5 \pm 3.8 & 0.14 \\ \text { Winter } & 1.1 \pm 2.0 & 0.08\end{array}$

$10.4 \pm 0.9 \quad 0.004$

\section{3}

$14.1-18.3$

7.5- 9.5

Summer

$0.7 \pm 1.4 \quad 0.03$

Winter

$0.6 \pm 1.1 \quad 0.02$

Summer $\quad 2.4 \pm 5.3 \quad 0.80$

Winter

$1.2 \pm 1.5 \quad 0.46$

14

08

(1)

$13.4 \pm 4.7 \quad 0.004 \pm$

0.002

$17.1 \pm 5.2 \quad 0.01 \pm 0.01$

$15.8 \pm 6.0 \quad 0.005 \pm$

0.002
Vermaat et al.

1993

Bandeira 1997

Bandeira 2000

0.18

de Boer 2000

0.18

0.62

0.20

0.20

0.08

$1.08 \pm 0.06$

Larsson 2009

0.93-1.98

Hanekom et al.

1988

$1.97 \pm 0.89$

Lyimo et al. 2006
$1.86 \pm 0.6$

$5.92 \pm 2.33$ 
In press with Botanica Marina, 2016

\begin{tabular}{|c|c|c|c|c|c|c|c|c|}
\hline & Marumbi & $6^{\circ} 13^{\prime} \mathrm{S}, 39^{\circ} 28^{\prime} \mathrm{E}$ & & $19.4 \pm 7.1$ & $0.02 \pm 0.01$ & & $2.05 \pm 0.9$ & \\
\hline & Chwaka & $6^{\circ} 10^{\prime} \mathrm{S}, 39^{\circ} 26^{\prime} \mathrm{E}$ & Enhalus acoroides & $24.8 \pm 9.4$ & $0.02 \pm 0.01$ & & $2.77 \pm 1.6$ & \\
\hline \multirow[t]{4}{*}{ Tunisia } & Ghar El Melh & $37^{\circ} 09^{\prime} \mathrm{N}, 10^{\circ} 13^{\prime} \mathrm{E}$ & Cymodocea nodosa & $3.35(21)$ & & $1.2 \pm 1(21)$ & $1.42(20)$ & Sghaier et al. 2011 \\
\hline & Lagoon & & & & & & & \\
\hline & Tabarka & $36^{\circ} 57^{\prime} \mathrm{N}, 8^{\circ} 45^{\prime} \mathrm{E}$ & Zostera noltii & & & 0.36 & & \\
\hline & El Kantaoui & $35^{\circ} 51^{\prime} \mathrm{N}, 10^{\circ} 35^{\prime} \mathrm{E}$ & Posidonia oceanica & & & 0.14 & & Sghaier et al. 2013 \\
\hline
\end{tabular}


118 Larger seagrass species such as Thalassia hemprichii and Thalassodendron ciliatum

119 recorded the highest per unit area biomass while smaller species, such as Halodule

120 wrightii, recorded the lowest biomass. There was a large range in biomass between the

121 highest and lowest species (Fig. 2).

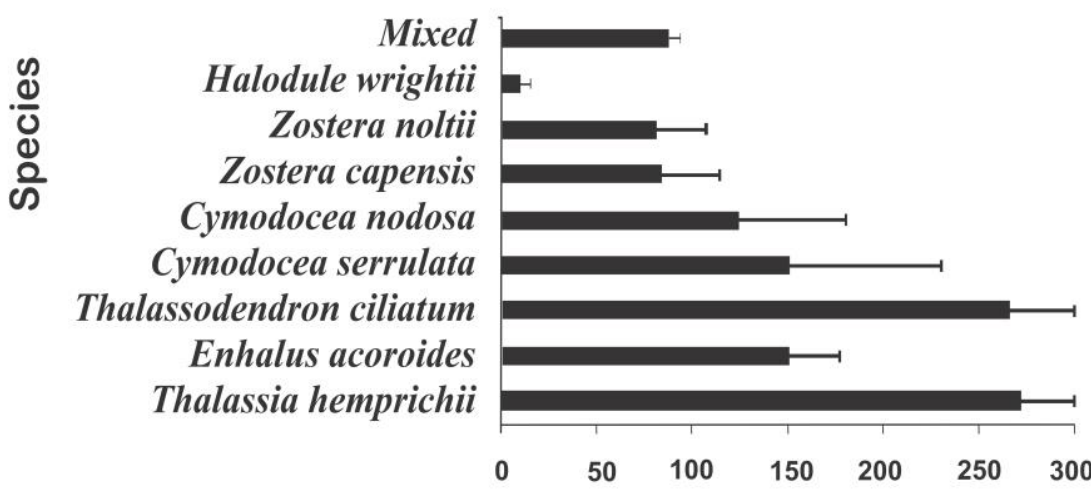

Above-ground-biomass (g DW m-2)
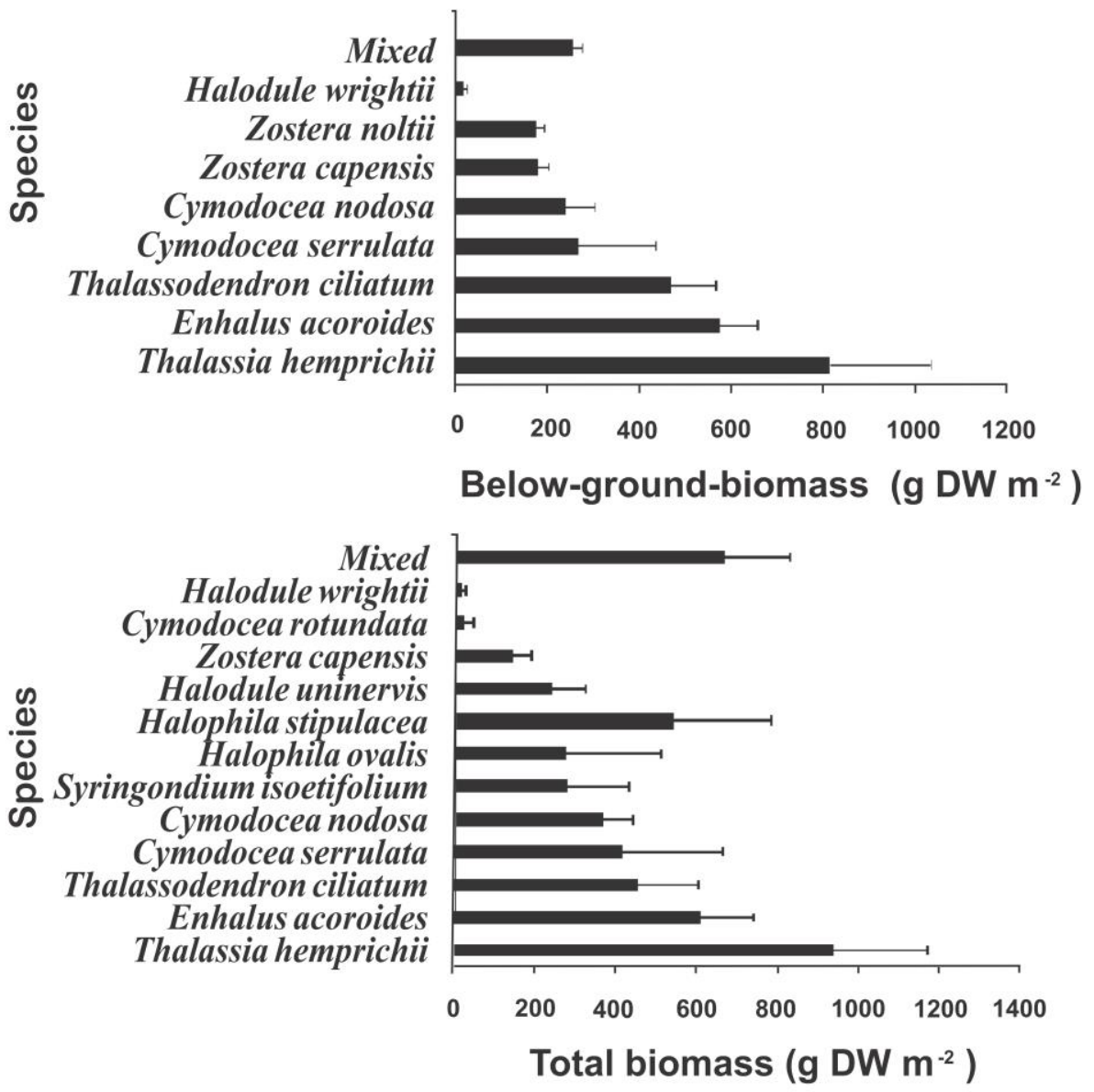

123 Fig. 2: Mean ( \pm S.E) above-ground, below-ground and total biomass values for 13 124 seagrass species studied in Africa, pooled across all reported sites 
125 The highest number of published biomass and productivity studies in Africa were

126 carried out between 1996 and 2010 accounting for $65.6 \%$ of the total, while $62.5 \%$ of

127 theses, reports or articles (unpublished or currently under peer review) have emerged

128 between 2010 and 2015 (Fig. 3).

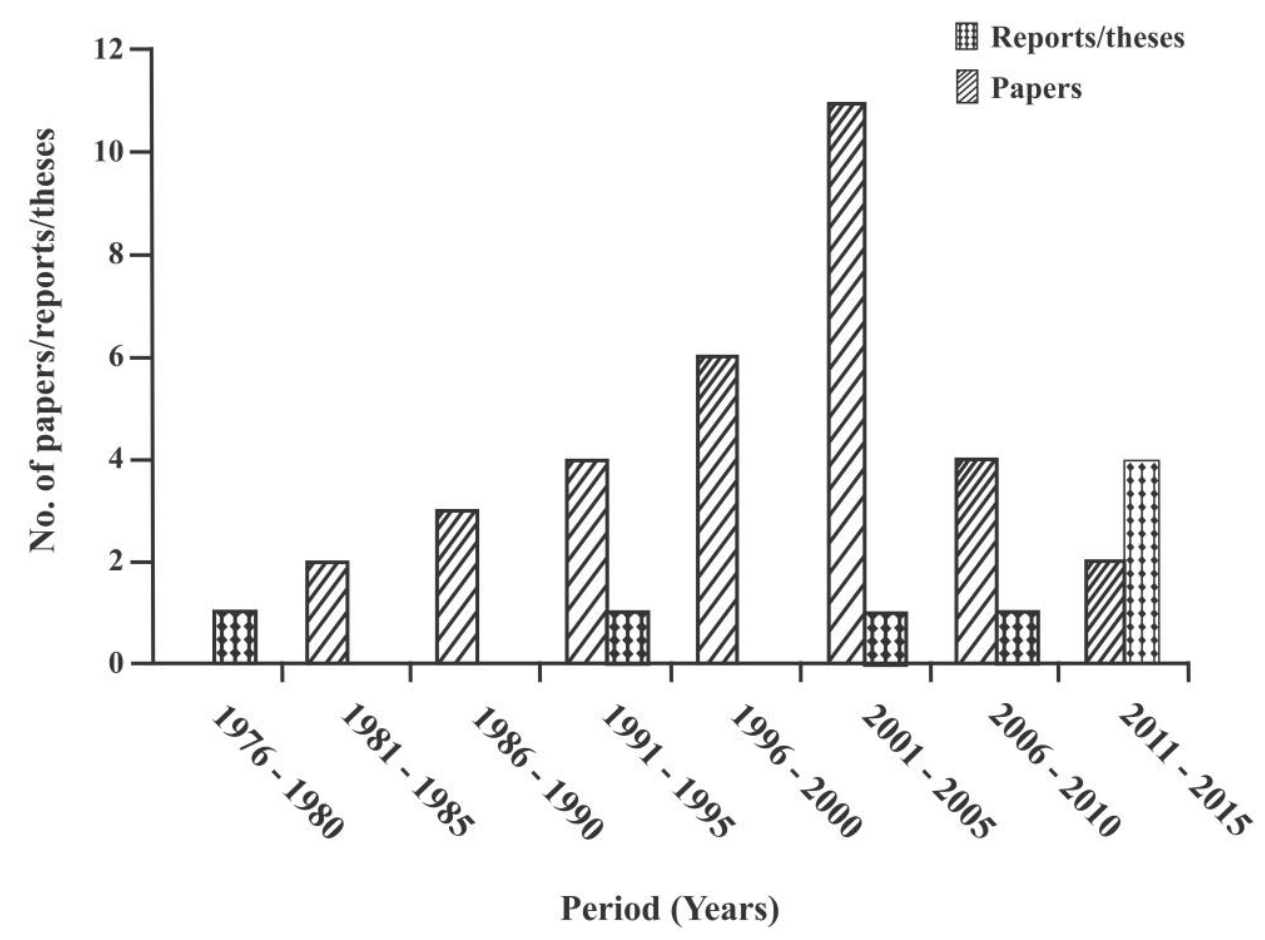

129

130 Fig. 3: Number of publications, reports/theses containing information on biomass and productivity of African seagrasses between 1976 and 2015

\section{Biomass of seagrasses in Africa}

134 We obtained 47 data sets for both the above- and below-ground biomass and 73 for total

135 biomass contained within the 32 papers and 8 reports or theses (Table 1). The total and

136 the above-ground biomass data were each reported in 21 of the 40 papers, reports and

137 theses while below-ground biomass was reported in 15 of those papers, theses and

138 reports. The total biomass for all species combined revealed large variation between 
139 sites (Table 2). The mean above- and below-ground biomasses for all species and across 140 all sites were 174.4 and $474.6 \mathrm{~g} \mathrm{DW} \mathrm{m}^{-2}$, respectively, representing an above to below-

141 ground biomass ratio of almost 1:3. The mean total biomass was $514.3 \mathrm{~g} \mathrm{DW} \mathrm{m}^{-2}$. This

142 was calculated from the data available on total biomass and not necessarily from the

143 sum of above-ground and below-ground biomass as some studies did not record either

144 the above-ground or the below-ground biomass (Table 2). The highest total biomass

145 was recorded for mixed seagrasses in a non-seaweed area at Jambiani in Zanzibar at

$1463063.3 \mathrm{~g} \mathrm{DW} \mathrm{m}^{-2}$ whilst the lowest total biomass of $0.6 \mathrm{~g} \mathrm{DW} \mathrm{m}^{-2}$ was recorded for

147 Halophila ovalis at Northern Bay on Inhaca Island off Mozambique in the same study

148 (Table 2). In terms of species, the highest biomass was recorded for Thalassia

149 hemprichii at $1876 \mathrm{~g} \mathrm{DW} \mathrm{m}^{-2}$ in Southern Bay of Inhaca Island, Mozambique (Table 2).

150 Comparison of the means for the above-ground, below-ground and total biomasses for

151 individual species reveal that the highest mean biomasses were found for Thalassia

152 hemprichii at $271.7 \mathrm{~g} \mathrm{DW} \mathrm{m}^{-2}, 817.8 \mathrm{~g} \mathrm{DW} \mathrm{m}^{-2}$ and $928.0 \mathrm{~g} \mathrm{DW} \mathrm{m}^{-2}$, respectively,

153 while the lowest mean biomasses were for Halodule wrightii at $11.5 \mathrm{~g} \mathrm{DW} \mathrm{m}^{-2}, 17.6 \mathrm{~g}$

$154 \mathrm{DW} \mathrm{m} \mathrm{m}^{-2}$ and $19.2 \mathrm{~g} \mathrm{DW} \mathrm{m}^{-2}$, respectively. In terms of the five regions where the

155 seagrass data are available (Fig. 4), the East African coast has the highest mean above-

156 ground, below-ground and total biomass at 256.8, 587.1 and $778.1 \mathrm{~g} \mathrm{DW} \mathrm{m}^{-2}$,

157 respectively. The South Mediterranean seagrasses had below-ground and above-ground

158 biomasses of 299.3 and $155.6 \mathrm{~g} \mathrm{DW} \mathrm{m}^{-2}$, respectively, while the South Africa and the

159 WIO Islands had means of 413.3 and $95.7 \mathrm{~g} \mathrm{DW} \mathrm{m}^{-2}$, respectively, for the same

160 parameters. Data available from the North West African region show the lowest mean

161 biomass for the three parameters with $61.06 \mathrm{~g} \mathrm{DW} \mathrm{m}^{-2}$ for the above-ground biomass,

$162145.2 \mathrm{~g} \mathrm{DW} \mathrm{m}^{-2}$ for the below-ground biomass and $159.4 \mathrm{~g} \mathrm{DW} \mathrm{m}^{-2}$ for the total

163 biomass (Fig. 4). 


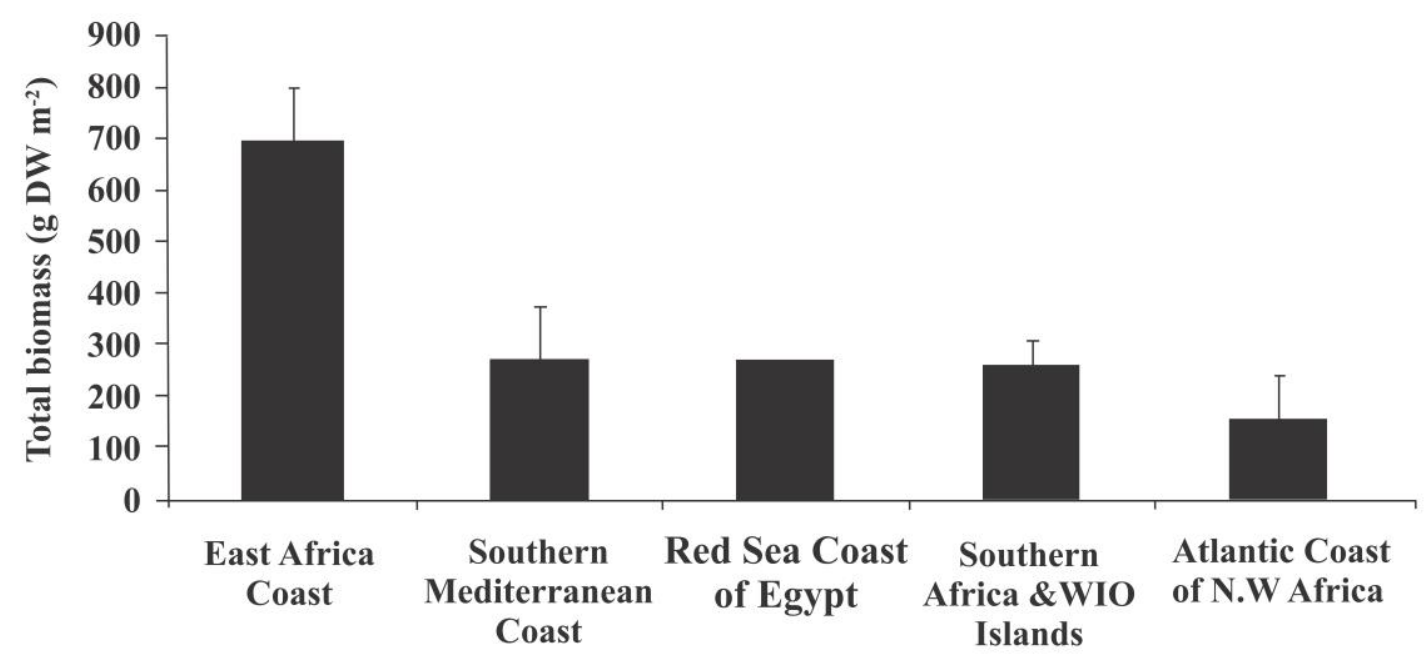

Region

Fig. 4: Mean $( \pm$ S.E) total biomass values for the seagrass species in different regions of Africa

\section{Productivity rates of seagrasses in Africa}

168 This review obtained 29 data sets on leaf growth rates, 24 on leaf production, 7 on rhizome growth rates and 32 on total production (Table 3). The mean leaf growth rate was $12.4 \mathrm{~mm}$ shoot $^{-1}$ day $^{-1}$ while the mean leaf production was $0.07 \mathrm{~g} \mathrm{DW}$ shoot $^{-1} \mathrm{~d}^{-1}$.

171 Rhizome growth rates were $0.36 \mathrm{~mm} \mathrm{~d}^{-1}$ while the mean total production was $2.5 \mathrm{~g} \mathrm{DW}$

172 shoot $^{-1} \mathrm{~d}^{-1}$. Lyimo et al. (2006) studied growth characteristics of Thalassia hemprichii

173 and Enhalus acoroides at several sites in Zanzibar, where high growth rates in terms of

174 leaf length and dry weight were observed for both species. In another study, Uku and

175 Bjork (2005) recorded higher growth rates for the same parameters for Thalassia

176 hemprichii as compared to Cymodocea rotundata and Thalassodendron ciliatum at

177 Nyali and Vipingo, Mombasa, Kenya. In Gazi Bay, Kenya, Hemminga et al. (1995) 
reported total productivity for Thalassodendron ciliatum that was much higher than reported from other sites (Table 3). In another study of a monospecific stand of Thalassodendron ciliatum at Gazi Bay, Ochieng (1995) recorded a mean shoot growth rate of $20.7 \mathrm{~mm} \mathrm{day}^{-1}$ which was higher than the rate recorded in most of the other studies for the same species. The review for all species, whether growing in multispecific or pure stands, indicated that Zostera capensis and Cymodocea serrulata had the lowest shoot growth rates of less than $1 \mathrm{~mm} \mathrm{shoot}^{-1}$ day $^{-1}$ recorded at Inhaca Island, Mozambique (de Boer, 2000). Some seasonality is indicated for Thalassia hemprichii with a maximum of $28.5 \mathrm{~mm} \operatorname{shoot}^{-1}$ day $^{-1}$ during the North East monsoon and 17.2 $\mathrm{mm} \mathrm{shoot}^{-1}$ day $^{-1}$ during the South East monsoon at Nyali in Mombasa (Uku and Björk. 2005). Daily leaf production also differed between sites and species with a maximum of $0.01 \mathrm{~g} \mathrm{DW}$ shoot $^{-1} \mathrm{~d}^{-1}$ for Thalassia hemprichii recorded at Chwaka in Zanzibar (Lyimo et al. 2006). Lowest daily leaf production was $0.001 \mathrm{~g} \mathrm{DW} \operatorname{shoot}^{-1} \mathrm{~d}^{-1}$ for Cymodocea rotundata recorded at Vipingo in Mombasa (Uku and Björk. 2005). The mean productivity rates for all species, where available, indicated that Thalassia hemprichii had the highest total productivity rates while the lowest was in an eelgrass, Zostera capensis (Table 4). The mean leaf production per day for individual species was highest in Cymodocea serrulata while the lowest was in Cymodocea rotundata. Comparison of rhizome growth rates indicated highest rates in Cymodocea nodosa and lowest in Posidonia oceanica. The mean for total production was highest in mixed stands while the lowest was recorded in Halophila ovalis (Table 4). 
This assessment of studies on seagrass biomass stocks and productivity around Africa found a limited number of papers and reports with most of them reporting from

202 countries on the Western Indian Ocean coastline (Kenya, Tanzania, Mozambique, South 203 Africa, Madagascar, Seychelles and Mauritius). A few studies have also been reported 204 from the Red Sea coastline of Egypt, the north eastern part of the Atlantic coastline on 205 the coast of Mauritania and Morocco and more recently some studies (unpublished), 206 have emerged from the Mediterranean coastline of Tunisia. However, the limited 207 number of studies demonstrates a paucity of information on the carbon budget and 208 flows in Africa. Similar observations of a geographical bias in research on seagrass 209 biomass stocks, with Africa particularly underrepresented, have been made in other 210 reviews (Duarte and Chiscano 1999, Fourqurean et al. 2012). Some of the seagrass 211 studies in Africa concentrated on one biomass pool (above-ground or below-ground) 212 while others focused on total biomass only (Table 2). An important observation in this 213 review is that seagrass studies in Africa have ignored the sediment organic carbon, the 214 most important part of the putative 'blue carbon' sink provided by seagrasses, revealing 215 a major gap in seagrass blue carbon work. Since the reviewed studies reported on only 21614 out of a total of 34 species in the Tropical Atlantic, Tropical Indo-Pacific and South 217 African flora, the current work suggests that the basic ecology, including productivity 218 and standing stock, of many species remains largely unknown.

219 The available data from the seagrass biomass and productivity studies in Africa reveal 220 that seagrasses allocate higher biomass to their below-ground than their above-ground components, with mean estimates for the above and below-ground biomasses of $174.4 \mathrm{~g}$

$222 \mathrm{DW} \mathrm{m} \mathrm{m}^{-2}$ and $474.6 \mathrm{~g} \mathrm{DW} \mathrm{m}^{-2}$, respectively. In a review of seagrass biomass from 
223 different studies globally, Duarte and Chiscano (1999) arrived at above- and below-

224 ground mean biomasses of $223.9 \mathrm{~g} \mathrm{DW} \mathrm{m}^{-2}$ and $237.4 \mathrm{~g} \mathrm{DW} \mathrm{m}^{-2}$, respectively. These

225 findings differ from the results of this study in which the above-ground biomass was

226 only $\sim 37 \%$ of the biomass below-ground. Though these results deviate from our

227 findings, our results are consistent with other observations, such as the most recent

228 review of a global dataset, that the below-ground component of seagrasses forms the

229 largest proportion of the living seagrass biomass and may constitute about two thirds of

230 the total biomass in seagrass meadows (Fourqurean et al. 2012). The similarity of

231 above-ground and below-ground biomass estimates in Duarte and Chiscano (1999) was

232 attributed to the fact that some seagrass biomass studies did not measure the below-

233 ground biomass, which in some cases could account for $15-50 \%$ of the total production

234 as observed in an earlier study (Duarte et al. 1998). Though grazing and mechanical

235 damage inflicted by wave scouring and by human activities may not significantly affect

236 seagrass productivity and biomass storage, it nevertheless impacts on the meadows

237 leading to high turnover rates especially for the above-ground component.

238 The mean estimate for total seagrass biomass in this review of $514.3 .4 \mathrm{~g} \mathrm{DW} \mathrm{m}^{-2}$ is

239 within the global range. The seagrasses of Abu Dhabi in the United Arab Emirates were

240 estimated to contain a total biomass of $122.3 \mathrm{~g} \mathrm{DW} \mathrm{m}^{-2}$ (Campbell et al. 2014). In a

241 review of global seagrass carbon storage, the Posidonia oceanica of the Mediterranean

242 Sea were found to have the highest biomass at $2144 \mathrm{~g} \mathrm{DW} \mathrm{m}^{-2}$ while the mean biomass

243 from the global seagrass data was estimated at $738.4 \mathrm{~g} \mathrm{DW} \mathrm{m}^{-2}$ (Fourqurean et al.

244 2012). While this global estimate is higher than our total African biomass estimate, this

245 could be explained by the influence of the high biomass of Posidonia oceanica in other

246 regions as well as the limited information on seagrass biomass from Africa in previous

247 global estimates. In terms of the five regions along the coasts of Africa where seagrass 
248 research has been done, this study observed that the East African seagrasses had the

249 highest biomass at $738.1 \mathrm{~g} \mathrm{DW} \mathrm{m}^{-2}$ compared to $370.8 \mathrm{~g} \mathrm{DW} \mathrm{m}^{-2}$ for the Southern

250 Mediterranean where Cymodocea nodosa was the dominant species. No study was

251 found from this southern part of the Mediterranean Sea containing information for

252 Posidonia oceanica.

253 The review observed that higher biomass values occurred in larger species compared to

254 the smaller species (Fig 2). This may suggest that larger species tend to develop higher

255 below-ground biomass and hence have a higher capacity for biomass storage due to the

256 relatively slow turnover of the below-ground materials (Duarte and Chiscano 1999).

257 The current assessment of available data from Africa on seagrass biomass supports this 258 view.

259 The current review arrived at a mean total production estimate of $912.5 \mathrm{~g} \mathrm{DW} \mathrm{m}^{-2} \mathrm{yr}^{-1}$

260 against $1012 \mathrm{~g} \mathrm{DW} \mathrm{m}^{-2} \mathrm{yr}^{-1}$ obtained in a previous seagrass biomass and production

261 reassessment using a global data set (Duarte and Chiscano 1999) and an earlier one of

$262816 \mathrm{~g} \mathrm{DW} \mathrm{m}^{-2} \mathrm{yr}^{-1}$ (Duarte and Cebrián 1996). Seagrass beds with mixed species were

263 found to have the highest total production, estimated at $1935 \mathrm{~g} \mathrm{DW} \mathrm{m}^{-2} \mathrm{yr}^{-1}$, followed

264 by Thalassodendron ciliatum at $1423 \mathrm{~g} \mathrm{DW} \mathrm{m}^{-2} \mathrm{yr}^{-1}$, suggesting that some species do

265 better when in association with others. Growth patterns for different species and

266 variation in environment between sites could account for the differences in values

267 observed. Some species may have the potential to accumulate biomass but this may be

268 kept low by resource limitation or due to the heavy losses caused by physical

269 disturbance (Duarte and Chiscano 1999). Biomass and productivity for some seagrass

270 species was reported to exhibit seasonality which could be attributed to periodical

271 fluctuations in abiotic factors such as irradiance, temperature and hydrological

272 conditions (Uku and Björk 2005,de Boer 2000). 
273 The estimates arrived at in this study may involve considerable errors, given the general

274 paucity of studies, particularly for some seagrass species, and a lack of uniformity in the

275 sampling methods used by different researchers. However, with the development of the

276 Blue Carbon sampling manual by the International Blue Carbon Initiatives Scientific

277 Working Group (Howard et al. 2014, http://the blue carbon initiative.org), and new

278 emphasis on researchers adopting uniform sampling protocols, future research should

279 produce more reliable and comparable estimates. Whilst the research gap revealed here

280 may be similar to many other areas in which Africa is under-represented, seagrasses

281 perhaps present a particular challenge for research in countries with relatively poor

282 infrastructure and resources, since they may require expensive sampling work utilizing

283 specialized skills such as scuba diving.

284 Considering that the African coastline is extensive with large areas of seagrass cover,

285 the spatial extent of study is very limited. The fact that this review did not find seagrass

286 biomass studies from the West African coast, with the exception of Mauritania which is

287 more to the North West coast, is another clear indication of the paucity of knowledge on

288 seagrass biomass stocks in Africa. A majority of the studies have been done on the West

289 Indian Ocean coastline mainly through funding by the West Indian Ocean Marine

290 Sciences Association (WIOMSA) in partnership with the well-established research

291 Institutions in the region or through partnership with institutions outside Africa. This

292 signifies the importance of strengthening collaboration between institutions and the

293 need for increased research funding if the knowledge gaps are to be filled. As the first

294 review of seagrass biomass and productivity in Africa, we hope the current work will

295 generate interest among the scientific community by identifying an important and

296 missed opportunity for research. By contributing to a better understanding of the role of 
297 seagrass ecosystems in carbon budgets in Africa this may help to support the protection 298 of these valuable ecosystems.

\section{Acknowledgements}

300 This article is based on research undertaken for Coastal Ecosystem Services in East 301 Africa (CESEA) NE/L001535/1 research project and was funded with support from the 302 Ecosystem Services for Poverty Alleviation (ESPA) programme. The ESPA programme 303 is funded by the Department for International Development (DFID), the Economic and 304 Social Research Council (ESRC) and the Natural Environment Research Council 305 (NERC). Additional support was made through Edinburgh Napier University and Kenya 306 Marine and Fisheries Research Institute (KMFRI) to which we are grateful. Finally, we 307 thank the three anonymous reviewers for their constructive comments which improved 308 the quality of the manuscript. 


\section{References}

310 Adams, J. B., and M. M. B. Talbot. 1992. The influence of river impoundment on the estuarine seagrass Zostera capensis Setchell. Bot. Mar.35: 69-76.

Aleem, A. A. 1984. Distribution and ecology of seagrass communities. Deep Sea Res.31: $919-933$.

Bandeira, S. O. 1997. Dynamics, biomass and total rhizome length of the seagrass Thalassodendron ciliatum at Inhaca Island, Mozambique. Plant Ecol.130: 133141.

Bandeira, S.O.2000. High production rates of the seagrass Thalassodendron ciliatum from the rocky and sandy habitats in Southern Mozambique: A comparative study In: (Diversity and Ecology of Seagrasses in Mozambique): Emphasis on Thalassodendron ciliatum. Structure, Dynamics, Nutrients and Genetic Variability. Ph. D. Thesis, Göteborg. University, Sweden. pp.18.

Bandeira, S. O. 2002. Leaf production rates of Thalassodendron ciliatum from rocky and sandy habitats. Aquat. Bot.72: 13-24.

Bououarour, O., R. EL.Kamcha, L. Boutahar, A. Tnoumi, Z. Bendahhou, A. Benhoussa and B. Hocein. 2015. In:(Mediterranean Seagrass Workshop). Spatial patterns of the Zostera noltii meadows across the Atlantic coast of Morocco: is there a latitudinal gradient?-Sardinia. Italy. pp. 81.

Boutahar, L., Y. S. Ramzi, O. Atef, R. EL. Kamcha, A. Benhoussa, E. Ostale and H. Bazairi. 2015. In (Mediterranean Seagrass Workshop).First data on biomass and 
In press with Botanica Marina, 2016

abundance of two eelgrass (Zostera marina) meadows south to the strait of Gilbraltar Jbel Moussa, Morocco. -Sardinia, Italy.pp. 109.

Campbell, J. E., E. A. Lacey, R. A. Decker, S. Crooks and J. W. Fourqurean. 2014. Carbon Storage in Seagrass Beds of Abu Dhabi, United Arab Emirates. Estuaries Coast. 38: 242-251.

Christie, N. D. 1981 - Primary production in the LangebaanLagoon. In:(J. H. Day. eds). Estuarine Ecology with Particular Reference to Southern Africa. Cape Town; Balkema. pp.101-115.

Daby, D. 2003. Effects of seagrass bed removal for tourism purposes in a Mauritian Bay. Environ. Pollut. 125: 313-324.

De Boer, W. F. 2000. Biomass dynamics of seagrasses and the role of mangrove and seagrass vegetation as different nutrient sources for an intertidal ecosystem. Aquat. Bot.66: 225-239.

Duarte, C. M. 1996a. Growth and population dynamics of Thalassodendron ciliatum in a Kenyan back-reef lagoon.Aquat. Bot.55: 1-11.

Duarte, C. M. and J. Cebrián.1996b. The fate of marine autotrophic production. Limnol. Oceanogr.41: 1758-1766.

Duarte, C., M. Merino and N. Agawin. 1998. Root production and below-ground seagrass biomass. Mar. Ecol.171: 97-108.

Duarte, C. and C. Chiscano. 1999. Seagrass biomass and production: a reassessment. Aquat. Bot.65: 159-174. 
Eklöf, J. S., M. de la Torre Castro, L. Adelsköld, N. S Jiddawi, and N. Kautsky. 2005. Differences in macrofaunal and seagrass assemblages in seagrass beds with and without seaweed farms. Est. Coast. Shelf Sci.63: 385-396.

Fourqurean, J. W.,C. M. Duarte, H. Kennedy, N. Marbà, M. Holmer, M. A. Mateo and D. Krause-jensen. 2012. Seagrass ecosystems as a globally significant carbon store. Nat. Geo.5: 1-5.

Gab-Alla, A. 2001. Ecological Status of the Seagrass Community in Sharm El-Moyia Bay (Gulf of Aqaba, Red Sea) after Oil Pollution in 1999. Mar. Sci.12: 231-239.

Grindley, J. R. 1976. Report on ecology of Knysna Estuaryand proposed Braamekraal Marina. (Internal Report). School of Environmental Studies, University of Cape Town. South Africa. pp.133.

Gullström, M., B. Lundén, M. Bodin, J. Kangwe, M. C. Öhman, M.S.P. Mtolera and M. Björk. 2006. Assessment of changes in the seagrass-dominated submerged vegetation of tropical Chwaka Bay (Zanzibar) using satellite remote sensing. Est. Coast. Shelf Sci.67: 399-408.

Gullström, M., M. Bodin, P. G. Nilsson and M.C. Öhman. 2008. Seagrass structural complexity and landscape configuration as determinants of tropical fish assemblage composition. Mar. Ecol. Prog. Ser.363:241-255.

Gwada, P. 2004. An assessment of seagrass survival and functioning in response to manipulations in sediment redox at Nyali Lagoon, MombasaKenya.WIOMSA report. MARG-1. GRANTEE 2001/2. 
372 Hanekom, N., L. Lagoon. 1988. Distribution and variations in seasonal biomass of eelgrass. Zostera capensis in the Kromme estuary, St Francis Bay South Africa. S.Afri. J. mar. Sci.7: 51-59.

Hemminga, M. A., P. Gwada, F. J. Slim, P. de Koeyer and J. Kazungu. 1995. Leaf production and nutrient contents of the seagrass Thalassodendron ciliatum in the proximity of a mangrove forest (Gazi Bay, Kenya). Aquat. Bot.50: 159-170.

Howard, J., S. Hoyt, K. Isensee, M. Telszewski and E. Pidgeon. 2014. (eds). Coastal Blue Carbon Methods for assessing carbon stocks and emmission factors in mangroves, tidal salt marshes and seagrasses. Conservation International, Intergovernmental Oceanographic Commission of UNESCO, International Union for Conservation of Nature, Arlington, Virginia, USA.

Imen, B. S.and S Abdessalem. 2015. In (Mediterranean Seagrass Workshop). Distribution, biomass and phenology of Zostera noltii meadows in the Northern lagoon of Tunis in the summer of 2014. -Sardinia, Italy. pp. 80.

Kamermans, P., M.A. Hemminga, J. F.Tack, M. A. Mateo, N. Marbà, M. Mtolera and Daele, T. Van. 2002. Groundwater effects on diversity and abundance of lagoonal seagrasses in Kenya and on Zanzibar Island ( East Africa ). Mar. Ecol. Prog. Ser.231: 75-83.

Laan, B. B., P. A. Van Der and W.J Wolff. 2006. Circular pools in the seagrass beds of the Banc d' Arguin, Mauritania, and their possible origin. Aquat. Bot.84: 93-100. 
Larsson, S. 2009. The Production of the Seagrass Thalassia hemprichii in relation to epiphytic biomass. M.sc. thesis (unpublished). Göteborg. University, Sweden. pp. 12.

Lugendo, B. R. Y. Mgaya and A. Semesi. 2001. The seagrass and associated macroalgae at selected beaches along Dar es Salaam coast. In: (M. D.Richmond and J.Francis, eds) Marine Science Development in Tanzania and East Africa. WIOMSA Books Series.pp. 359-374.

Lyimo, T. J.,E.F. Mvungi, C. Lugomela and M. Björk. 2006. Seagrass biomass and productivity in Seaweed and Non- Seaweed Farming areas in the East Coast of Zanzibar.West Indi. Ocea. J. Mar. Sci.5: $141-152$.

Lyimo, T., E. Mvungi and Y. Mgaya. 2008. Abundance and diversity of seagrass and macrofauna in the intertidal areas with and without seaweed farming activities in the east coast of Zanzibar. Tanz. J. Sci.34:41-52.

Martins, A. and S. Bandeira. 2001. Biomass distribution and leaf nutrient concentrations and resorption of Thalassia hemprichii at Inhaca Island, Mozambique. S. Afri. J. Bot.67: 439-442

Mateo M.A., J. Cebrian, K. Dunton, T. Mutchler 2006. Carbon Flux in Seagrass Ecosystem. In: (A. Larkum, R. Orth and C. Duarte. eds). Seagrass Biology, Ecology and Conservation. Netherlands, Springer-Verlag. pp.159-192.

Mostafa, H.M. 1996. Preliminary observations of the seagrass Cymodocea nodosa (Ucria) Ascherson in the Mediterranean waters of Alexandria, Egypt. Bull. of Natl Inst. Ocea. Fish. 22: 19-28. 
Mvungi, E. F. 2011. Seagrasses and eutrophication Interactions between seagrass photosynthesis. Ph.Dthesis (unpublished)Stockholm University, Sweden. pp 44.

Nellemann, C., E. Corcoran, C. M. Duarte, L.Valdes, C. De Young, L. Fonseca and Grimsditch. (Eds).2009. Blue carbon. A Rapid Response Assessment.GRIDArendal.United Nations Environmental Programme. ISBN: 978-82-7701-060-1.

Ochieng, C. A. 1995. Productivity of seagrasses with respect to intersystem fluxes Gazi Bay (Kenya). In: Interlinkages between Eastern African Coastal Ecosystems. Contract No .T53-CT92-0114. Final report. pp. 82-86.

Ochieng, C. A.and P. L. Erftemeijer.1999. Accumulation of seagrass beach cast along the Kenyan coast: a quantitative assessment. Aquat. Bot.65:221-238.

Ochieng, C. A. and P. L. Erftemeijer.2003. Seagrasses of Tanzania and Kenya..In: (E. Green, F. Short, eds). World Atlas of Seagrasses. World Conservation Monitoring Centre. University of California Press, USA, Berkely. pp. 93-100.

Paula, J., P. F. Ecosta, A. Martins and D. Gove.2001. Patterns of abundance of seagrasses and associated infaunal communities at Inhaca Island, Mozambique. Est. Coast. Shelf Sci. 53: 307-318.

Pergent, G.,A. Djellouli,A. A. Hamza, K. S. Ettayeb, A. A. El Mansouri, F. M. Talha, and F Platini.2002. Characterization of the benthic vegetation in the Farwà Lagoon (Libya). J. Coast. Cons. 8: 119-126.

Semroud, R., S.Mezegrane, L.Soltane. 1990. Etude lépidochronologique de Posidonia oceanica dans la régiond'Alger (Algérie): donnéspréliminaires. Rapp. P.V.Réun. Comm.Int. Explor. Sci. Médit. 31: 10. 
Sghaier, Y. R., R. Zakhama-Sraieb, and F. Charfi-Cheikhrouh.2011. Primary production and biomass in a Cymodocea nodosa meadow in the Ghar El Melh lagoon, Tunisia. Bot. Mar.54: 411-418.

Sghaier, Y. R. 2012. Seasonal variation of Cymodocea nodosa in the Ghar El Melh lagoon ( Tunisia ), with reference to insolation, temperature and salinity effects.Bull. Inst. Natn. Scien. Tech.Mede. Salammbõ. 39: 117-125.

Sghaier, Y. R., R. Zakhama-Sraieb, and F. Charfi-Cheikhrouha.2013. Patterns of shallow seagrass (Posidonia oceanica) growth and flowering along the Tunisian coast. Aquat. Bot.104: 185-192.

Short, F., T. Carruthers,W. Dennison, and M. Waycott. 2007. Global seagrass distribution and diversity: A bioregional model. J. Exp. Mar. Biol. and Ecol.350: $3-20$.

Talbot, M. M. B., G. C. Bate,. 1987. The distribution and biomass of the seagrass Zostera capensis in a warm-temperate estuary. Bot. Mar.30: 91-99.

Uku, J. N., E. E. Martens and K. M. Mavuti. 1996. An Ecological Assessment of Littoral Seagrass Communities in Diani and Galu Coastal Beaches, Kenya.M.sc. thesis (unpublished). University of Nairobi. pp 185.

Uku, J. N and Björk, M. 2005. Productivity aspects of three tropical seagrass species in areas of different nutrient levels in Kenya. Est. Coast. Shelf Sci63: 407-420.

Van Lent, F., P. H. Nienhuis, and J.M Verschuure. 1991. Production and Biomass of the Seagrasses Zostera-noltii Hornem and Cymodocea nodosa (Ucria) Aschers at the 
Banc-Darguin (Mauritania, N.W Africa). A Preliminary approach. Aquat. Bot.41:

458 $353-367$.

Vermaat, J. E., J. J. Beijer,R. Gijlstra, M. J. M. Hootsmans, C. J. M. Philippart., N. W. van den Brink, and W.van Vierssen. 1993. Leaf dynamics and standing stocks of intertidal Zostera noltii Hornem. and Cymodocea nodosa (Ucria) Ascherson on the Banc d'Arguin (Mauritania). Hydrobiologia.258: 59-72. 